Programa de Pós-Graduação em Engenharia de Produção - PPGEP

Laboratório de Qualidade de Vida - LaQVida

Universidade Tecnológica Federal do Paraná - UTFPR

Ponta Grossa - PR - Brasil

v. 01 , n. 02 , jul./dez. 2009 , p. $25-38$

DOI: $10.3895 / \mathrm{S} 2175-08582009000200004$

\author{
REVISTA BRASILEIRA DE \\ QUALIDADE DE VIDA
}

\title{
Análise espacial intra-urbana da qualidade de vida em Curitiba
}

\section{Spatial analysis of the intra-urban life quality in Curitiba}

\author{
Wagner Hugo Bonat \\ Result Consultoria em Avaliação e Laboratório de Estatística e Geoinformação - LEG/UFPR - Curitiba - Brasil \\ wbonat@gmail.com \\ Maria de Fátima Paiva \\ Instituto de Pesquisa e Planejamento Urbano de Curitiba - IPPUC - Curitiba - Brasil \\ mariaf@ippuc.org.br \\ Regina Maria Sliwiany \\ Result Consultoria em Avaliação - Curitiba - Brasil \\ result@result.com.br
}

\begin{abstract}
Resumo
A prática do planejamento urbano com base em métodos consistentes é hoje uma demanda para os grandes centros urbanos do país. Neste sentido o uso adequado de técnicas estatísticas para dados espaciais se faz necessário para subsidiar a tomada das decisões governamentais com bases mais objetivas. Um dos grandes desafios atuais é a compreensão da dimensão espacial dos processos sociais, assim como a dependência desses processos dentro do espaço urbano. Neste artigo é apresentada uma abordagem metodológica baseada no Índice de Qualidade de Vida de Curitiba (IQVC), com o qual através de índices de autocorrelação espacial, foi possível dividir a cidade em grupos homogêneos, indicando onde as melhores e piores condições de vida ocorrem, assim como também definir áreas prioritárias para a intervenção levando-se em conta o efeito da dependência espacial detectada. Tais resultados são de fundamental importância, em um processo de planejamento que visa diminuir os grandes contrastes sociais, dentro do limite urbano.
\end{abstract}

Palavras-chave: índice de qualidade de vida de Curitiba, índices de autocorrelação espacial, planejamento urbano.

\begin{abstract}
The practice urban planning with basis on consistent methods, it's today a demand for the great urban centers of the country. In this aspect the proper use of statistics techniques to spatial data is made necessary to subsidize the governmental decision taking with an objective foundation. One of the great challenges today it is the comprehension of the space dimension of social processes, just like the dependence of this processes in the urban area. In this paper is presented a methodological approach based on the Curitiba's Quality of Life Indicator, which one, though the indicator of space autocorrelation, made possibility to split the city in homogeneous groups, indicating where the bests and worst life conditions occur, also like define areas that have priority in this matter to the intervention taking into account the detected space dependence effects. These results are of fundamental importance in a planning process that sights to decrease the great social contrasts, inside the urban limit.
\end{abstract}

Keywords: Curitiba's quality of life indicator, autocorrelation spatial indicators, urban planning. 


\section{Introdução}

Um dos maiores desafios para todos aqueles que estudam os fenômenos sociais, econômicos e ambientais é a necessidade de combinar a compreensão histórica e qualitativa desses processos com o tratamento quantitativo de dados, para subsidiar a tomada de decisões em bases mais objetivas. Neste contexto compreender a distribuição espacial dos fenômenos sociais constitui hoje um desafio para a elucidação de questões importantes referentes ao planejamento urbano da cidade (DRUCK et al., 2005).

Tais estudos estão se tornando cada vez mais comuns, devido à disponibilidade de Sistemas de Informações Geográficas (SIG's) de baixo custo e com interfaces amigáveis, nesta área pode-se citar, por exemplo, a plataforma Terralib e um de seus subprodutos o TerraView, ambos desenvolvidos pelo Instituto Nacional de Pesquisas Espaciais (INPE), e distribuídos na forma de software livre e com código aberto (CÂMARA et al., 2000). Estes sistemas permitem a apresentação espacial de variáveis diversas como, densidades populacionais, índices de qualidade de vida, ou mesmo vendas de uma empresa em uma região por meio de mapas coloridos. Este tipo de uso dos SIG's permite uma percepção visual da distribuição espacial da variável em análise, porém além desta percepção é muito útil traduzir padrões existentes no espaço em considerações objetivas e mensuráveis, a análise espacial nos permite ir além dos mapas coloridos, e estabelecer uma quantificação explicita da variabilidade espacial do fenômeno em estudo. Segundo, Câmara (2004),

\footnotetext{
Não basta acreditar na primeira lei da Geografia (todas as coisas se parecem mais coisas mais próximas são mais parecidas que as mais distantes). É preciso modelar a distribuição do "parentesco" entre os dados, por meio de técnicas de Estatística Espacial. Com isto, poderemos ter maior grau de confiabilidade em nossas investigações e em nosso entendimento dos problemas de gestão de nossos recursos naturais e socioeconômicos (CÂMARA, 2004, p. 2).
}

Entre os inúmeros conceitos de estatística espacial sem dúvida um conceito chave na compreensão dos fenômenos espaciais é o da dependência espacial, Cressie (1991) comenta que a dependência espacial está presente em todas as direções e fica mais fraca à medida que aumenta a dispersão na localização dos dados.

Em geral a maior parte dos fenômenos sociais, apresentam entre si uma relação que depende da distância, por exemplo, se encontramos na cidade um bairro com condições educacionais extremamente ruins, espera-se que os bairros vizinhos a este apresentem uma situação semelhante.

Estudos têm mostrado que a heterogeneidade social, ambiental e econômica, proporciona efeitos espaciais diversos, este conceito vem sendo definido pela ausência de estabilidade no espaço, nos modelos espaciais, as formas funcionais dos parâmetros variarão com a localização geográfica da variável. Como exemplo de tais estudos pode-se citar, Sposati (1996) a qual construiu um Mapa da Exclusão/Inclusão social da cidade de São Paulo, Haddad e Nedovic-Budic (2006) que realizou um estudo examinando as desigualdades intraurbanas em São Paulo, e Peréz (2005) que realizou um estudo sobre a dimensão espacial da pobreza na Colômbia.

Este problema da heterogeneidade espacial tem sido discutido em estudos sobre as economias regionais, e também dentro do ambiente de planejamento urbano e políticas sociais. Nos últimos anos uma série de métodos estatísticos para dados espaciais, vêm sendo desenvolvidos, tais métodos permitem avaliar a existência de relações sociais e econômicas entre diversos fatores intervenientes, como também, a relação destes com a sua localização geográfica (HENNINGER; SNEL, 2002). Assim o propósito principal tem sido estabelecer a existência ou não da dependência espacial entre as áreas geográficas estudadas (PETRUCCI; SALVATI; SEGHIERI, 2004).

A distribuição espacial dos fenômenos sociais tem sido um dos aspectos que tem gerado grande preocupação em vários países e regiões principalmente entre os sub desenvolvidos e os em via do desenvolvimento. Anselin (1988) ao falar da pobreza, refere-se ao fato de que a população pobre encontra-se concentrada em algumas áreas geográficas específicas, encontrar essas áreas e 
direcionar as políticas de atenção social a elas, é hoje sem dúvida um avanço nos métodos de planejamento social e políticas públicas.

O objetivo ao utilizar este tipo de ferramenta é acrescentar a visão clara e específica dos problemas de cada área geográfica, com a finalidade de permitir aos formuladores de políticas sociais executar de forma eficiente os projetos de desenvolvimento. Além disso, permite mostrar de forma simples, informações aos não-especialistas no tema, os quais conseguem examinar facilmente informações cartográficas para identificar clusters, além de tendências e padrões espaciais.

Em Curitiba, a Análise Exploratória para dados Espaciais (ESDA) (ANSELIN; BAO, 1997), será aplicada como ferramenta para análise de diferenciais intra-urbanos, tendo como base de estudo o índice de qualidade de vida de Curitiba (IQVC). Será estudada uma série de indicadores sociais os quais compõe o IQVC, com o objetivo de detectar semelhanças e diferenças espaciais, identificar clusters de bairros que se autocorrelacionam fortemente, descobrir padrões de associações espaciais, sugerir regimes espaciais ou outras formas de heterogeneidade espacial, buscando assim, identificar os bairros prioritários para as políticas públicas que visam melhorar a qualidade de vida da população curitibana, assim como, identificar situações e bairros mais abastados que serviram como metas a serem alcançadas com a implementação das políticas sociais, dando também um inicio ao processo de avaliação de políticas sociais, prática essa não comum, entre as cidades brasileiras. Procura-se também estabelecer a importância do uso de técnicas espaciais, que possam contribuir significativamente para a formulação e implementação de políticas para a redução da desigualdade social ao nível intra-urbano medido pelos indicadores de qualidade de vida do IQVC.

\section{Medindo a qualidade de vida}

O Instituto de Pesquisa e Planejamento Urbano de Curitiba (IPPUC) foi o primeiro no Brasil, em 1980, a aplicar avaliações da qualidade de vida de sua população que auxiliam o plano diretor da cidade e seu planejamento urbano a curto, médio e longo prazo.

O IQVC é composto por cinco grandes áreas de necessidades sociais (habitação, saúde, educação, segurança e transporte), representadas por indicadores qualitativos norteadores de ações locais, baseadas em diferenciais intraurbanos. Tem por objetivo, consolidar um processo de monitoramento das condições de vida da população com ênfase em seis pontos:

1. Comparação entre os indicadores encontrados na cidade e em cada um dos seus 75 bairros com as melhores e piores situações encontradas, seja dentro da própria cidade, seja em outras cidades, regiões ou países;

2. Avaliação constante da qualidade de vida do município como um todo e dos diferentes bairros, (micro-áreas), de maneira a observar sua evolução;

3. Caracterização dos diferenciais intraurbanos da cidade, estabelecendo-se índices de condições de vida para cada bairro, permitindo comparação entre eles;

4. Disseminação das informações analíticas dos resultados globais ou locais nas áreas especificas para que sejam estipulados processos de transformações das diferentes realidades locais;

5. Indicação de bairros que necessitem maior intervenção do poder púbico com o objetivo de reduzir as desigualdades;

6. Oferecer subsídios ao planejamento urbano e a criação de políticas especificas para o município, auxiliando na priorização das ações e na tomada de decisões gerenciais, informando, inclusive a sua localização geográfica.

O IQVC é construído pelo Método Distancial ou Genebrino (SLIWIANY, 1997), o qual mensura, basicamente, os resultados dos benefícios sociais alcançados por uma população. Estatisticamente, admite-se o nível de vida como o estado atual das condições concretas de vida e não uma condição desejada ou esperada. Nesse contexto Sliwiany (1997) define que 
culturais das economias domesticas $\left(\mathrm{Y}_{1 \mathrm{td}}, \mathrm{Y}_{2 \mathrm{td}}, \ldots, \mathrm{Y}_{\mathrm{k}-\mathrm{ltd}}, \mathrm{Y}_{\mathrm{ktd}}\right)$ obtido no sentido da garantia dessa satisfação, através dos fluxos de mercadorias e de serviços pagos e dos fluxos do fundo de consumos coletivo (SLIWIANY, 1997, p. 25)

A utilização do Método Distancial ou Genebrino permite análises que podem ir do geral ao especifico e vice-versa, através da construção dos índices:

- Índice Sintético: determina o nível de crescimento global de todos os grupos de necessidades sociais avaliados;

- Índices Grupais: mostram o crescimento de cada um dos setores avaliados (habitação, saúde, educação, segurança e transporte);

- Índices Parciais: apresentam os resultados do alcance dos benefícios sociais para cada um dos indicadores utilizados em cada um dos setores.

Para a análise deste índice, tradicionalmente são feitos dois tipos de análise; a multicêntrica e a intraurbana. Apesar deste trabalho ter como foco a análise intraurbana, vai-se decorrer de forma ampla sobre a análise multicêntrica.

$\mathrm{Na}$ análise multicêntrica são comparados os graus de satisfação das necessidades, a partir do estabelecimento de parâmetros externos à cidade, ou seja, os limiares mínimos e máximo que foram estabelecidos considerando-se os padrões de qualidade existente em todo o país. Para os setores de habitação, saúde, educação e segurança, os parâmetros utilizados na analise multicêntrica foram obtidos a partir de valores encontrados nas capitais brasileiras. Devido a peculiaridades do Sistema de Transporte Coletivo em Curitiba, e da dificuldade de comparação com outras cidades brasileiras, optou-se por excluir este setor da analise multicêntrica.

$\mathrm{Na}$ análise intraurbana, base deste trabalho, são examinados os padrões de qualidade existente dentro da própria cidade, utilizando-se o critério lógico estatístico (SLIWIANY, 1997) a partir dos valores encontrados nos bairros da cidade. Esta análise permite a identificação dos espaços urbanos onde as desigualdades se manifestam, instrumentalizando o planejamento.

O quadro 1, apresenta o elenco de indicadores que compõe o IQVC, os valores mínimos e máximos utilizados como parâmetros pelo método Distancial ou Genebrino, e o resultado de todos os índices (Parciais, Grupais e Sintético), para o bairro Centro, este foi escolhido, já que, a analise intraurbana não gera um índice para a cidade como um todo, apenas para cada um de seus 75 bairros.

Neste ponto é interessante deixar claro que a medida utilizada em todo trabalho é o correspondente ao índice global se fará referencia a este como o índice de Qualidade de Vida, sendo que cada um dos 75 bairros tem o seu próprio índice, e este resume as condições dos bairros com relação a todos os setores sociais avaliados. 
Quadro 1: Índice de Qualidade de Vida nos setores de habitação, saúde, educação, segurança e transporte - Bairro Centro Curitiba - 2000

\begin{tabular}{|c|c|c|c|c|c|c|c|}
\hline Setores / Indicadores & $\begin{array}{l}\text { Leitura } \\
\text { dos } \\
\text { Dados }\end{array}$ & $\begin{array}{c}\text { Valor } \\
\text { Mínimo }\end{array}$ & $\begin{array}{c}\text { Valor } \\
\text { Empírico }\end{array}$ & $\begin{array}{c}\text { Valor } \\
\text { Máximo }\end{array}$ & $\begin{array}{l}\text { Índice } \\
\text { Parcial }\end{array}$ & $\begin{array}{l}\text { Índice } \\
\text { Grupal }\end{array}$ & $\begin{array}{c}\text { Índice } \\
\text { Sintético }\end{array}$ \\
\hline \multirow{3}{*}{$\begin{array}{l}\text { 1. Habitação } \\
\text { 1.1 Domicílios em aglomeradas subnormais } \\
\text { 1.2 Domicílios com rede de esgoto ou fossa } \\
\text { séptica }\end{array}$} & \multirow{3}{*}{$\begin{array}{l}\% \\
\%\end{array}$} & \multirow{3}{*}{$\begin{array}{l}13.72 \\
82.31\end{array}$} & \multirow{3}{*}{$\begin{array}{c}0 \\
99.92\end{array}$} & \multirow{3}{*}{$\begin{array}{c}0.02 \\
98.72\end{array}$} & \multicolumn{3}{|c|}{$0 \%-100 \%$} \\
\hline & & & & & 100 & \multirow[b]{2}{*}{100} & \multirow{20}{*}{64.16} \\
\hline & & & & & 100 & & \\
\hline \multicolumn{8}{|l|}{ 2. Saúde } \\
\hline 2.1 Mortalidade infantil & $/ 1.000$ & 18.13 & 19.16 & 9.88 & 0 & & \\
\hline 2.2 Baixo peso ao nascer $(<2.5 \mathrm{Kg})$ & $\%$ & 9.59 & 9.31 & 7.05 & 11.24 & & \\
\hline 2.3 Mortalidade geral padronizada & $/ 1.000$ & 6.51 & 6.74 & 3.41 & 0 & & \\
\hline 2.4 Incidência de tuberculose & $/ 100.000$ & 56.95 & 71.73 & 21.32 & 0 & 15.89 & \\
\hline 2.5 Incidência de AIDS & $/ 100.000$ & 57.11 & 194.34 & 24.28 & 0 & & \\
\hline 2.6 Mortalidade $<$ de 5 anos & $/ 1.000$ & 19.79 & 21.12 & 11.69 & 0 & & \\
\hline 2.7 Hepatite A & $/ 100.000$ & 71.81 & 17.78 & 18.51 & 100 & & \\
\hline 3. Educação & & & & & & & \\
\hline 3.1 Taxa de reprovação & $\%$ & 12.06 & 5.04 & 3.41 & 81.12 & & \\
\hline 3.2 Taxa de abandono & $\%$ & 4.66 & 0.80 & 0.34 & 89.29 & 90.14 & \\
\hline 3.3 Taxa de analfabetismo & $\%$ & 5.64 & 0.66 & 0.91 & 100 & & \\
\hline 4. Segurança & & & & & & \multirow{7}{*}{14.77} & \\
\hline 4.1 Homicídios & $/ 1.000$ & 0.24 & 0.17 & 0.04 & 36.63 & & \\
\hline 4.2 Acidentes de trânsito & $\%$ & 1.96 & 7.11 & 0.36 & 0 & & \\
\hline 4.3 Procedência do adolescente infrator & $\%$ & 2.38 & 1.59 & 0.26 & 37.21 & & \\
\hline 4.4 Ocorrências policiais & $/ 10.000$ & 2021.96 & 8920.24 & 873.75 & 0 & & \\
\hline 4.5 Mortalidade por causas externas & $/ 10.000$ & 7.19 & 7.66 & 3.05 & 0 & & \\
\hline $\begin{array}{l}\text { 5. Transporte } \\
5.1 \text { Linhas ligadas a RIT }\end{array}$ & $\mathrm{N}^{\mathrm{o}}$ & 5.74 & 40 & 20.69 & 100 & & \\
\hline
\end{tabular}

Fonte: Instituto de Pesquisa e Planejamento Urbano de Curitiba (2004)

\section{Metodologia estatística}

Este estudo utiliza como unidade de medida os bairros da cidade de Curitiba, os quais possibilitaram utilizar técnicas de análise exploratória de dados espaciais (ESDA), para estabelecer se a Qualidade de Vida, dos bairros de Curitiba se distribui de forma aleatória ou, se pelo contrário, existe evidência de dependência espacial.

Este conjunto de ferramentas é definido na literatura como sendo uma coleção de técnicas, para descrever e visualizar distribuições espaciais, identificar situações atípicas, descobrir padrões de associação espacial, agrupamento de valores semelhantes (clusters) e sugerir regimes espaciais ou outras formas de heterogeneidade espacial (ANSELIN; BAO, 1997).

O procedimento inicial para a implementação de tais técnicas, é importar para o software especifico, tanto as informações vetoriais, que descrevem os limites de cada bairro, como também seus atributos alfanuméricos, lembrando que cada polígono representa um bairro, e a esse polígono é associado seus indicadores representantes das necessidades sociais de cada bairro (atributos alfanuméricos).

Em todas as técnicas de ESDA empregadas neste estudo, estão presente três elementos básicos: a matriz de proximidade espacial (W), o vetor de desvios (Z) e o vetor de médias ponderadas $(\mathrm{Wz})$. A apresentação das técnicas baseadas neste três elementos básicos foi baseada em Neves et al. (2000).

A matriz de proximidade espacial, é uma ferramenta geral e bastante útil para descrever o arranjo espacial dos objetos (BAILEY; GATRELL, 1995). O critério utilizado para a construção desta matriz (W) foi o de adjacência ou contigüidade vários critérios podem ser usados para isso, uma discussão sobre essas matrizes pode ser encontrada em Oliver e Gotway (2005), o critério utilizado é definido da seguinte forma:

$$
\text { Bairros com fronteira comum, } W_{i j}=1 \text {; }
$$


Bairros sem fronteira comum, $W_{i j}=0$.

Para o vetor dos desvios $(Z)$, é calculado primeiramente a média $(\mu)$, dos valores dos atributos $\left(y_{i}\right)$, considerando os $n$ objetos. Cada valor de i de $\mathrm{Z}$ é obtido da seguinte forma: $Z_{i}=y_{i}-\mu$.

O vetor das médias ponderadas (Wz), é obtido pela multiplicação do vetor transposto dos desvios, pela matriz de proximidade espacial com linhas normalizadas.

Dados estes três elementos, o índice de Moran fornece uma medida geral da associação espacial (autocorrelação) presente nos dados. Este índice de uma forma geral presta-se a um teste de hipótese, cuja hipótese nula é a de independência espacial, assumindo neste caso o valor zero, valores positivos ( 0 a 1) indicam autocorrelação direta (positiva), e valores negativos ( -1 a 0 ), correlação inversa (negativa). Representando o índice de Moran em função dos três elementos básicos descritos, tem-se a seguinte expressão:

$$
I=\frac{Z^{t} W_{z}}{Z^{t} Z}
$$

onde $Z^{t}$ é o vetor de desvios transposto.

Uma das formas de se verificar a significância estatística do índice de Moran obtido, é através de um teste chamado de pseudodistribuição, neste caso, são geradas diferentes permutações dos valores dos atributos associados às regiões; cada permutação produz um novo arranjo espacial, no qual os valores são redistribuídos entre as áreas. Como apenas um dos arranjos corresponde à situação observada, pode-se construir uma distribuição empírica para o índice de Moran, se o valor do índice encontrado corresponder a um extremo da distribuição simulada, então trata-se de valor com significância estatística (DRUCK et al. 2005).

Uma maneira adicional e muito útil de visualizar a dependência espacial é o Diagrama de Espalhamento de Moran, este diagrama é apresentado na figura 1.

Figura 1 - Diagrama de Espalhamento de Moran

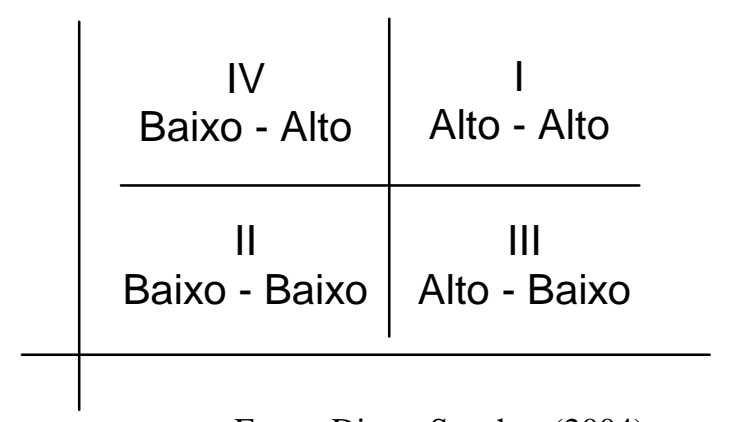

Fonte: Diaz e Sanches (2004)

Os quadrantes são interpretados da seguinte forma:

Q1 - valores positivos, médias positivas; Q2 - valores negativos, médias negativas. Indicam pontos de associação espacial positivos, levando-se em conta que em uma mesma localidade há vizinhos (bairros) com valores semelhantes.

Q3 - valores positivos, médias negativas; Q4 - valores negativos, médias positivas. Indicam pontos de associação espacial negativos, considerando que em uma mesma localização há vizinhos (bairros) com valores distintos (DRUCK et al., 2005).

Como está se lidando com um grande número de áreas, é muito provável que diferentes regimes de autocorrelação espacial ocorram dentro desta área. Para evidenciar estes regimes utilizou-se indicadores de associação espacial, que podem ser associados a diferentes localizações da variável. O índice local utilizado neste estudo, foi o índice local de Moran, uma das formas de representação deste índice, em função dos elementos básicos é: 


$$
I_{i}=\frac{z_{i} W_{z}}{\sigma^{2}}
$$

onde

$I_{i}$ : índice local para o bairro $\mathrm{i}$;

$Z_{i}$ : valor do desvio do bairro $\mathrm{i}$;

$W z_{i}$ : valor médio dos desvios dos bairros vizinhos do bairro i.

$\sigma^{2}$ : variância da distribuição dos valores dos desvios.

\section{Resultados}

Em primeiro lugar avaliou-se a existência de dependência espacial no índice de Qualidade de Vida de Curitiba, para isso utilizou-se a estatística “ I de Moran”, testando a hipótese nula de não existência de dependência espacial. Este índice informa o nível global da autocorrelação espacial entre todas as regiões, ou seja, 75 bairros da cidade de Curitiba.

O índice global de Moran medido foi de 0.4282, uma pseudodistribuição foi gerada com 999 valores, esta indicou um p-valor de 0.001, o que leva a rejeitar a hipótese nula (não autocorrelação entre os bairros), com uma significância de $99.9 \%$. Pode-se dizer então que a Qualidade de Vida em Curitiba apresenta evidências de forte estrutura de autocorrelação espacial: parte variação ampla, ou tendência, parte dependência espacial entre vizinhos.

O índice de Moran é equivalente ao coeficiente de regressão linear que indica a inclinação da reta de regressão. Isto significa, que ao aumentar um ponto no índice de qualidade de vida de um bairro, este impacta em um aumento de 0.4282 na média do índice de qualidade de vida da sua vizinhança.

Como dito anteriormente, uma forma adicional e eficiente de visualizar esta dependência espacial é através do Diagrama de Espalhamento de Moran, a figura 2 apresenta este diagrama para o caso do índice de Qualidade de Vida de Curitiba.

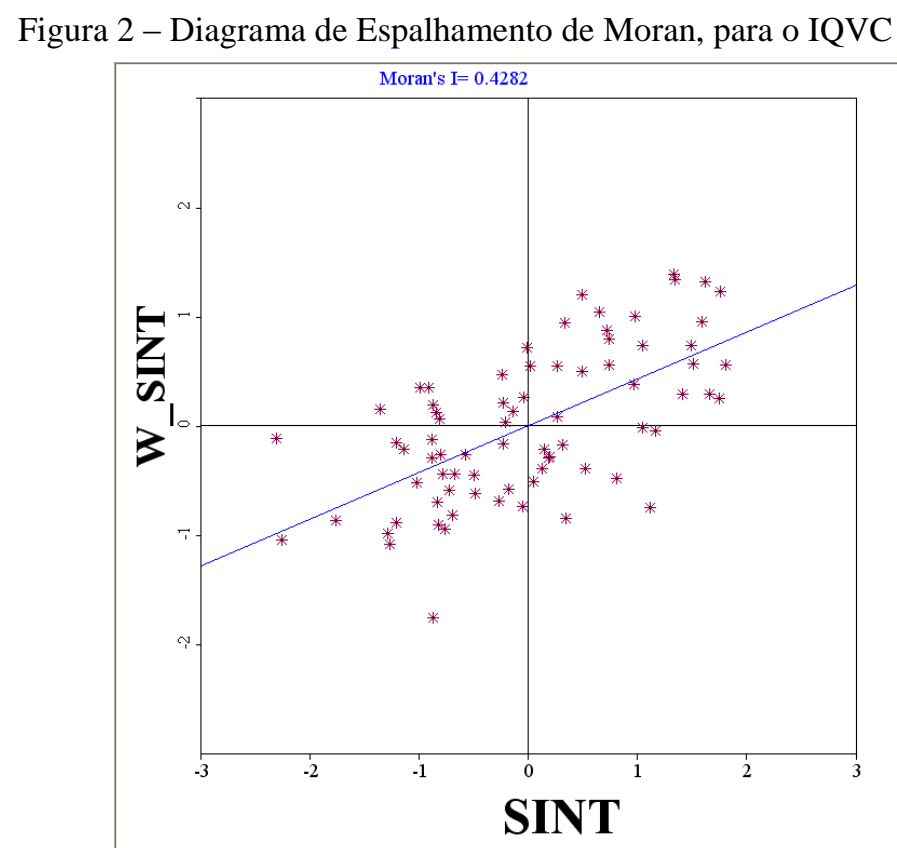

Fonte: Autoria própria (2009)

O resultado do diagrama, comprova a significância do índice de Moran, mostrando que a maioria dos pontos estão localizados nos quadrantes 1 e 2 . Os pontos localizados nos quadrantes $3 \mathrm{e}$ 4 podem ser vistos como regiões que não seguem o mesmo processo de dependência espacial, 
evidentemente o diagrama reflete a estrutura espacial nas duas escalas de análise: vizinhança e tendência.

O diagrama de espalhamento de Moran também pode ser apresentado por meio de um mapa bidimensional onde os bairros são agrupados pela posição que ocupam no diagrama, este mapa (1) é apresentado na medida do IQVC, indica respectivamente os quadrantes Q1 “Alto-Alto", Q2 "BaixoBaixo", Q3" Alto-Baixo" e Q4 "Baixo-Alto".

Nota-se na figura 3, forte polarização de regiões em azul "Alto-Alto", que são bairros localizados na região central e norte do município. As áreas em verde "Alto-Baixo" são bairros de transição entre a região central e a região sul, o que mostra claramente que os bairros mais próximos do centro da cidade tendem a ter melhor qualidade de vida, do que bairros mais afastados. Os bairros em amarelo "Baixo-Alto" podem ser visto com áreas atípicas e também uma zona de transição, pois são bairros que apresentam baixa qualidade de vida, porém estão rodeados de bairros com alta qualidade de vida. Nota-se também, que as regiões em vermelho são predominantes no mapa, abrangendo os bairros localizados em toda a região sul e oeste de Curitiba, esta é a região onde se encontra a população mais carente da cidade.

Figura 3 - Representação do Diagrama de Espalhamento de Moran, para o IQVC - 2000

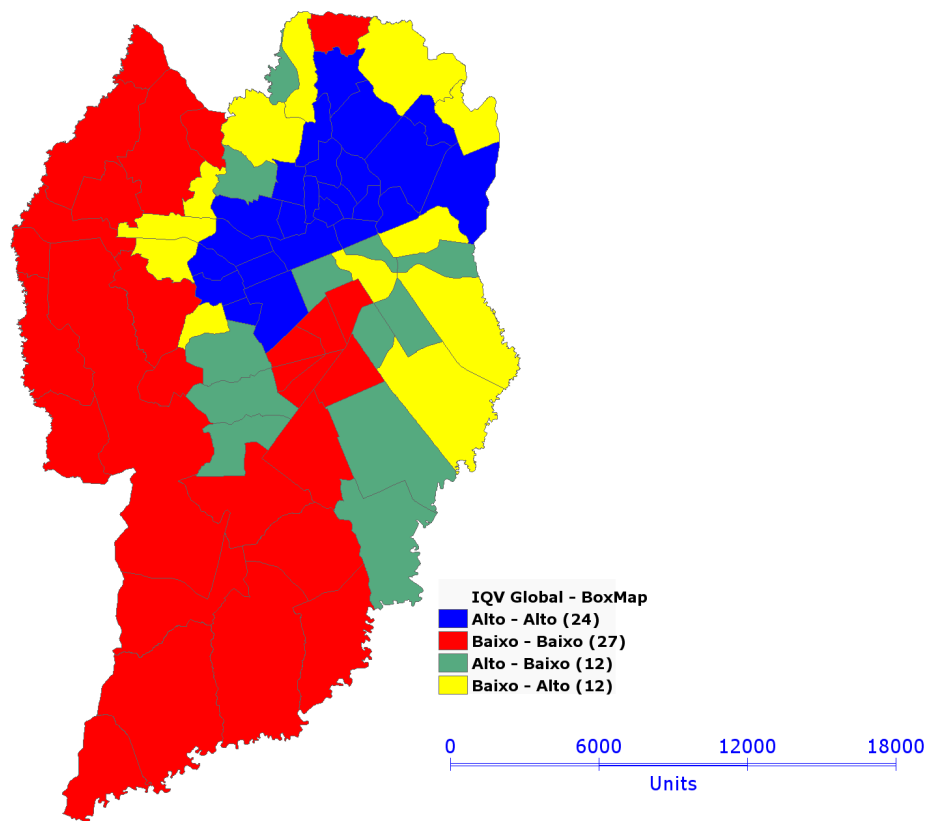

Fonte: Autoria própria (2009)

Com base neste mapa, pode-se construir uma classificação intraurbana da Qualidade de Vida em Curitiba, que leva em consideração explicitamente à estrutura espacial em que esta se desenvolve. Neste sentido classificaram-se os bairros da cidade em quatro categorias (Alta, Baixa, Média-Alta e Média) de acordo com os quatro quadrantes do diagrama de espalhamento de Moran.

A vizinhança tida como de Alta Qualidade de Vida (azul), é composta por 24 bairros, apresenta um índice médio de 76.3, o desvio padrão é de 9.6 resultando em um coeficiente de variação de 0.10 mostrando uma grande homogeneidade dentro deste cluster, mostrando que as políticas sociais voltadas para esta área da cidade devem ser semelhantes. Este cluster também pode ser visto como meta para os demais. Como ele apresenta as melhores condições dentro da cidade, é de interesse que todos os outros bairros, atinjam tais indicadores, e também é uma meta factível de ser atingida, já que, ela existe na realidade intra-municipal. Este cluster abriga 364.113 habitantes segundo o Censo 2000, e representa aproximadamente $23 \%$ da população de Curitiba, conforme tabela a seguir. 
Tabela 1 - Vizinhança de Alta Qualidade de Vida - Curitiba 2000

\begin{tabular}{llclcr}
\hline \multicolumn{1}{c}{ Bairros } & IQV & Habitantes & \multicolumn{1}{c}{ Bairros } & IQV & Habitantes \\
\hline Água Verde & 87.03 & 49.866 & Campina do Siqueira & 71.11 & 7.108 \\
Ahú & 88.78 & 11.148 & Centro & 64.16 & 32.623 \\
Alto da Glória & 66.95 & 5.588 & Centro Cívico & 85.80 & 4.767 \\
Alto da Rua XV & 84.55 & 8.683 & Hugo Lange & 81.56 & 3.167 \\
Bacacheri & 84.23 & 23.106 & Jardim Social & 70.83 & 6.085 \\
Bairro Alto & 58.79 & 42.033 & Juvevê & 81.40 & 11.281 \\
Barreirinha & 62.91 & 17.021 & Mercês & 63.03 & 14.089 \\
Batel & 75.21 & 11.778 & São Francisco & 76.44 & 6.435 \\
Bigorrilho & 88.56 & 27.127 & São Lourenço & 82.83 & 5.556 \\
Boa Vista & 69.60 & 29.391 & Seminário & 66.85 & 7.395 \\
Bom Retiro & 71.12 & 5.633 & Tingui & 75.14 & 11.564 \\
Cabral & 86.34 & 11.720 & Vila Izabel & 89.60 & 10.949 \\
\hline
\end{tabular}

A vizinhança de piores condições de vida (vermelho), é composta de 27 bairros, apresenta um índice médio de 42.7 com um desvio padrão de 9.4 resultando em um coeficiente de variação de 0.22 , também mostra grande homogeneidade dentro do cluster, porém menor que a do anterior. Esta vizinhança é a mais carente da cidade, devendo ser a área prioritária de intervenção das políticas sociais para melhorar suas condições de vida. O cluster abriga 629.941 habitantes, que representam aproximadamente $40 \%$ da população residente em Curitiba, conforme tabela abaixo.

Tabela 2 - Vizinhança de Baixa Qualidade de Vida - Curitiba 2000

\begin{tabular}{lcclcc}
\hline \multicolumn{1}{c}{ Bairros } & IQV & Habitantes & \multicolumn{1}{c}{ Bairros } & IQV & Habitantes \\
\hline Augusta & 44.25 & 3.617 & Lindóia & 44.94 & 8.343 \\
Butiatuvinha & 57.54 & 10.759 & Orleans & 40.72 & 7.260 \\
Cachoeira & 48.45 & 7.738 & Parolin & 18.57 & 11.982 \\
Campo Comprido & 49.95 & 21.638 & Pinheirinho & 45.85 & 49.689 \\
Campo de Santana & 43.26 & 7.335 & Prado Velho & 37.62 & 7.084 \\
Caximba & 27.93 & 2.475 & Rivieira & 37.50 & 203 \\
Cidade Industrial & 44.08 & 157.461 & Santa Felicidade & 49.92 & 25.209 \\
Fanny & 53.70 & 7.866 & São Braz & 46.83 & 23.119 \\
Fazendinha & 38.69 & 26.122 & São João & 43.22 & 2.950 \\
Ganchinho & 36.24 & 7.325 & São Miguel & 46.50 & 4.911 \\
Guaíra & 43.11 & 14.268 & Sítio Cercado & 45.18 & 102.410 \\
Hauer & 55.34 & 13.851 & Tatuquara & 36.52 & 36.339 \\
Lamenha Pequena & 44.48 & 701 & Umbará & 19.44 & 14.595 \\
& & & Xaxim & 54.43 & 54.691 \\
\hline
\end{tabular}

Fonte: Fundação Instituto Brasileiro de Geografia e Estatística (2001)

A vizinhança classificada como de Média-Alta Qualidade de Vida (verde), é composta por 12 bairros, apresenta um índice médio de $67.0 \mathrm{com}$ um desvio padrão de 7.2, resultando em um coeficiente de variação de 0.10 . Como nas anteriores mostra homogeneidade dentro do cluster.Esta vizinhança pode ser vista como uma zona de transição entre a vizinhança de piores condições, em direção ao cluster de altas condições. Ela abriga 324.921 habitantes, que representam aproximadamente $20 \%$ da população residente em Curitiba, conforme tabela abaixo. 
Tabela 3 - Vizinhança de Média-Alta Qualidade de Vida - Curitiba 2000

\begin{tabular}{llllll}
\hline \multicolumn{1}{c}{ Bairro } & IQV & Habitantes & \multicolumn{1}{c}{ Bairros } & IQV & Habitantes \\
\hline Alto Boqueirão & 59.25 & 51.155 & Jardim das Américas & 77.69 & 13.966 \\
Boqueirão & 61.79 & 68.495 & Portão & 76.50 & 40.735 \\
Capão da Imbuia & 60.96 & 20.976 & Rebouças & 72.42 & 15.618 \\
Capão Raso & 60.52 & 34.376 & Taboão & 64.42 & 2.668 \\
Cristo Rei & 78.61 & 13.325 & Novo Mundo & 61.62 & 42.999 \\
Guabirotuba & 67.49 & 10.678 & Vista Alegre & 63.89 & 9.930 \\
\hline
\end{tabular}

Fonte: Fundação Instituto Brasileiro de Geografia e Estatística (2001)

A vizinhança classificada como de Média Qualidade de Vida (amarelo), é composta por 12 bairros, apresenta um índice médio de 48.8 com um desvio padrão de 7.8 resultando em um coeficiente de variação de 0.16 , mostrando homogeneidade dentro do cluster. Como dito anteriormente, esta vizinhança pode ser vista como uma área de transição, esta vizinhança abriga 268.340 habitantes que representam aproximadamente $17 \%$ da população de Curitiba, conforme tabela a seguir.

Tabela 4 - Vizinhança de Média Qualidade de Vida - Curitiba 2000

\begin{tabular}{lcclcc}
\hline \multicolumn{1}{c}{ Bairros } & IQV & Habitantes & \multicolumn{1}{c}{ Bairros } & IQV & Habitantes \\
\hline Abranches & 43.89 & 11.165 & Pilarzinho & 43.37 & 27.907 \\
Atuba & 57.62 & 12.632 & Tarumã & 58.19 & 7.045 \\
Cajuru & 34.90 & 89.784 & Santa Cândida & 54.31 & 27.870 \\
Cascatinha & 55.98 & 2.061 & Santa Quitéria & 41.25 & 11.720 \\
Jardim Botânico & 42.68 & 6.153 & Santo Inácio & 54.81 & 6.037 \\
Mossungue & 54.51 & 5.628 & Uberaba & 44.33 & 60.338 \\
\hline
\end{tabular}

Fonte: Fundação Instituto Brasileiro de Geografia e Estatística (2001)

É interessante fazer uma comparação entre as vizinhanças obtidas, para verificar o nível de desigualdade intraurbana nas condições de vida. Verifica-se que enquanto a vizinhança de Alta Qualidade de Vida apresenta um índice médio de 76.3 a vizinhança de baixa Qualidade de Vida apresenta um índice médio de 42.7, ou seja, 33.6 unidades a menos que o cluster de alta condições de vida, além disso verifica-se ainda um agravante, a vizinhança de alta condições de vida abriga aproximadamente $23 \%$ da população enquanto que a de baixa abriga aproximadamente $40 \%$ da população.

Esses resultados mostram uma grande desigualdade no acesso as condições de vida no nível intramunicipal. Na zona de transição tem-se aproximadamente $37 \%$ da população, sendo que a diferença entre as médias desses dois clusters é de 18.2 unidades, esses resultados evidenciam a desigualdade intraurbana no nível de Qualidade de Vida em Curitiba. A tabela 5 apresenta as principais medidas descritivas para cada um dos 4 clusters obtidos e também com a média de todos os bairros da cidade.

Tabela 5 - Comparação entre os clusters globais, com a média dos bairros de Curitiba-2000

\begin{tabular}{lccccc}
\hline \multicolumn{1}{c}{ Estatísticas } & & Média-Alta & Média & Baixa & $\begin{array}{c}\text { Média dos } \\
\text { Bairros }\end{array}$ \\
\hline Média & 76.37 & 67.10 & 48.82 & 42.75 & 58.36 \\
Des. Padrão & 9.63 & 7.25 & 7.84 & 9.46 & 17.28 \\
Coef. Variação & 0.12 & 0.10 & 0.16 & 0.22 & 0.29 \\
Habitantes & 364.113 & 324.921 & 268.340 & 629.941 & 1.587 .315 \\
\% Curitiba & 22.9 & 20.4 & 16.9 & 39.6 & 100 \\
\hline
\end{tabular}

Fonte: Autoria própria (2009)

Apesar do diagrama de espalhamento de Moran, dar uma classificação intraurbana que leva em consideração o espaço, e o índice global de Moran, trazer uma medida da dependência espacial, ainda é interessante detectar pontos de associação espacial onde a autocorrelação é ainda mais 
pronunciada, para subsidiar ações mais pontuais em localizações estratégicas, sabendo que uma ação nestas áreas terá um efeito de difusão para as áreas vizinhas, ainda mais forte do que o medido com o índice global de Moran. Para detectar essas áreas foi utilizado o índice local de Moran, as regiões onde a autocorrelação espacial é significativamente diferente das demais podem ser vistas como "bolsões" de não-estacionariedade, pois são áreas com dinâmica espacial própria e merecem análise detalhada.

Esta ferramenta possibilita ao planejamento interpretar de forma mais simples e direta como os dados estão distribuídos no espaço, possibilitando, assim, definir intervenções mais pontuadas, visando eliminar mais rapidamente as desigualdades sociais existentes entre as regiões da cidade.

A aplicação do índice local de Moran, para o IQVC 2000, é apresentada, por um mapa bidimensional (figura 4), este evidencia dois clusters, o primeiro corresponde aos bairros que apresentam alto Índice de Qualidade de Vida (azul) e estão na vizinhança de bairros com a mesma situação. Este cluster pode ser visto como a área de maior Qualidade de Vida da cidade, e que se autocorrelacionam fortemente, ela compreende 13 (Ahú, Alto da Glória, Alto da Rua XV, Bacacheri, Batel, Boa Vista, Bom Retiro, Cabral, Centro, Centro Cívico, Hugo Lange, Jardim Social e Juvevê) bairros que abrigam 164.970 habitantes, os quais representam aproximadamente $10 \%$ da população de Curitiba, o índice médio é de 77.7 , o desvio padrão é de 8.3 , resultando em um coeficiente de variação de 0.10 , o que mostra a homogeneidade do cluster.

O segundo cluster corresponde aos bairros de piores condições de vida, que se autocorrelacionam fortemente, é a área da cidade de maior carência em suas necessidades básicas, necessitando de forte intervenção governamental. Este cluster é composto por 7 (Campo de Santana, Cidade Industrial, Fanny, Ganchinho, Sitío Cercado, Tatuquara e Umbará), bairros que abrigam 333.331 habitantes os quais representam aproximadamente $21 \%$ da população de Curitiba, o índice médio é de 39.7, o desvio padrão é de 10.7 resultando em um coeficiente de variação de 0.26, o que mostra uma homogeneidade interna, porém menor que a encontrada no primeiro cluster.

Figura 2 - Representação do Índice Local de Moran para o IQVC - 2000

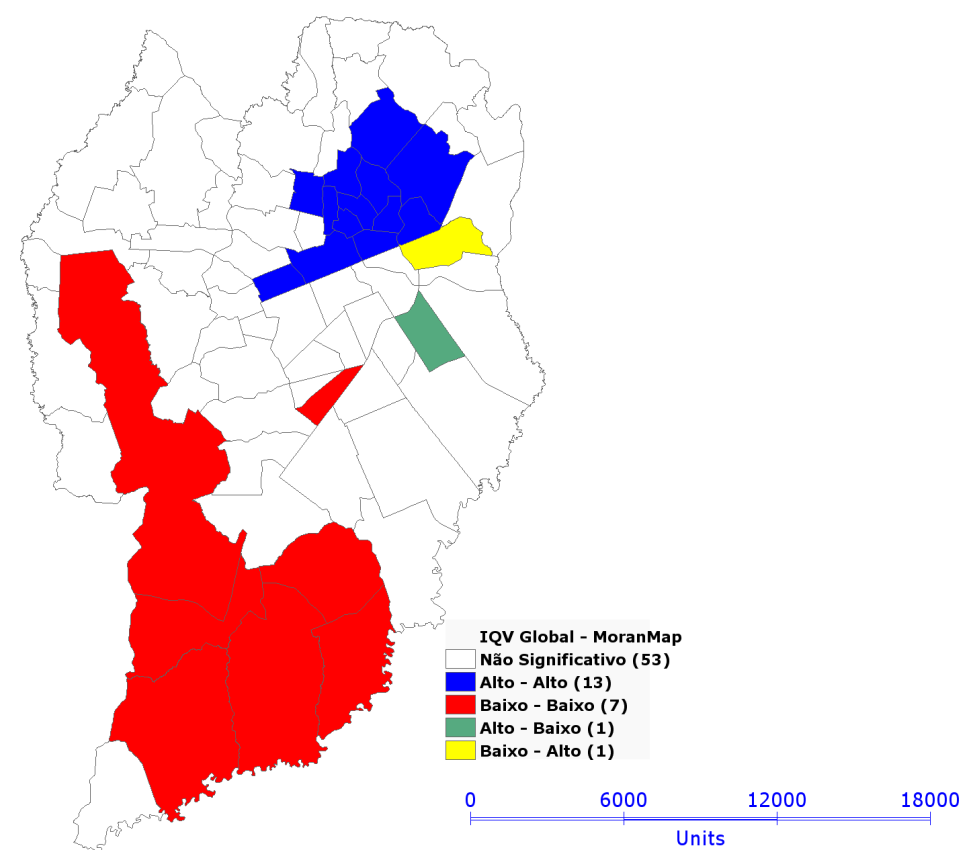

Fonte: Autoria própria (2009)

Comparando os clusters tem-se que a média do cluster 1 é 77.7, a média do cluster 2 é de apenas 39.7, ou seja, 37.9 unidades a menos que o cluster de maior desenvolvimento, e 18.5 unidades a menos que a média da cidade, existe ainda um agravante o cluster 2, conta com aproximadamente $21 \%$ da população de Curitiba, enquanto que o cluster 1, conta com apenas $10 \%$ dessa população, isso demonstra um alto grau de desigualdade social dentro do limite municipal, já 
que apenas 10\% da população vive em uma vizinhança de alta Qualidade de Vida. A tabela 6 faz a comparação entre os dois clusters obtidos pelo índice local de Moran.

Tabela 6 - Comparação dos Clusters Locais, com a média dos bairros de Curitiba 2000

\begin{tabular}{lccc}
\hline \multicolumn{1}{c}{ Estatísticas } & $\begin{array}{c}\text { Cluster 1 (azul) } \\
\text { (Alto- Alto) }\end{array}$ & $\begin{array}{c}\text { Cluster 2 (vermelho) } \\
\text { (Baixo- Baixo) }\end{array}$ & Média dos Bairros \\
\hline Média Aritmética & 77.73 & 39.77 & 58.36 \\
Desvio Padrão & 8.37 & 10.72 & 17.28 \\
Coeficiente de Variação & 0.107 & 0.26 & 0.29 \\
População & 164.970 & 333.331 & 1.587 .315 \\
\% Curitiba & $10.39 \%$ & $20.99 \%$ & $100 \%$ \\
Bairros & 13 & 07 & - \\
\hline
\end{tabular}

Fonte: Autoria própria (2009)

\section{Conclusão}

Num cenário em que a avaliação e o monitoramento da Qualidade de Vida, servem de instrumento para subsidiar o planejamento urbano da cidade, buscou-se neste trabalho, um aprimoramento técnico baseado em métodos estatísticos que permitiram mensurar o grau da autocorrelação espacial entre os 75 bairros da cidade de Curitiba, para tanto utilizou-se a Análise Exploratória de Dados Espaciais, a qual apresenta um conjunto de ferramentas e técnicas que permitiram visualizar relações de dependência espacial existentes entre os 75 bairros da cidade. Tais técnicas podem ampliar consideravelmente a capacidade de compreender padrões espaciais associados a dados de área, especialmente quando se trata de indicadores sociais que apresentam autocorrelação tanto global como local.

Em termos gerais a análise espacial de dados fornece mais subsídios para a tomada de decisão, acrescentando a visão espacial do fenômeno em estudo, indicando qual a área que deve ter prioridade e áreas onde a melhor situação ocorre. Enfim, ao separar uma cidade em grupos homogêneos internamente, pode-se detectar pelas similaridades espaciais as precariedades nas condições de vida.

Com relação aos resultados da aplicação dos métodos estatísticos, em primeiro lugar pode-se afirmar que existe evidência significativa da dependência espacial entre os bairros da cidade na medida do Índice de Qualidade de Vida, assim, é possível afirmar que a localização geográfica tem papel fundamental na determinação do nível de Qualidade de Vida nos bairros de Curitiba, como também, que a Qualidade de Vida de um bairro depende de forma significativa da Qualidade de Vida de seus bairros vizinhos.

O segundo resultado importante, é a identificação do cluster de baixa Qualidade de Vida de bairros que se autocorrelacionam fortemente, já que, este é o cluster prioritário para as políticas sociais, sendo que para este grupo é necessário um esforço adicional para superar esta situação de insatisfação de suas necessidades sociais básicas.

Além disso, quantificou-se o efeito que um bairro tem sobre a sua vizinhança, por exemplo, se uma ação é feita em determinado bairro, e eleva o índice de Qualidade de Vida em uma unidade, tem-se que o efeito médio esperado de aumento nos vizinhos é de 0.4282 .

Dentro da perspectiva de se mapear a Qualidade de Vida dos bairros da cidade de Curitiba, estas ferramentas estatísticas mostraram grande valia, pois possibilitaram uma compreensão mais coerente da dinâmica espacial, permitindo observar regimes espaciais extremamente polarizados, dentro do limite urbano, evidenciando o paradoxo que vive os grandes centros urbanos do país.

Deixa-se aqui como futuras agendas de pesquisa o fato de analisar quais os fatores que contribuem para o aumento da Qualidade de Vida na cidade de Curitiba, ou seja, quais são os meios que determinam maior ou menor Qualidade de Vida em determinado bairro, além disso, considerase importante quantificar o impacto dos equipamentos urbanos na Qualidade de Vida da população. 


\section{Referências}

ANSELIN, L. Spatial econometrics. Boston: Kluwer Academic, 1988.

ANSELIN, L.; BAO, S. Exploratory Spatial data Analysis Linking SpaceStat and ArcView. In: FISCHER, M.M.; GETIS, A. Recent developments in spatial analysis. New York: Springer, 1997. p. 35-59.

BAILEY, T. C.; GATRELL, A. C. Interactive spatial data analysis. Harlow: Longman, 1995.

CÂMARA, G. et al. Terralib: Technology in Support of GIS Innovation. In: SIMPÓSIO BRASILEIRO EM GEOINFORMÁTICA, 2., 2000, São Paulo. Anais... São Paulo: GEOINFO, 2000.

CÂMARA, G. Análise espacial: além dos mapas coloridos, 2004. Disponível em: 〈http://www.dpi.inpe.br/gilberto/infogeo/infogeo8.pdf>. Acesso em: 25 fev. 2009.

CRESSIE, N. Statistics for spatial data analysis. New Iork: Wiley, 1991.

DIAZ, A. M.; SÁNCHEZ, F. Geografia de los cultivos ilícitos y conflicto armado em Colômbia, 2004. Disponível em < http://ideas.repec.org/p/col/000089/002765.html>. Acesso em: 04 jan. 2010.

DRUCK, S. et al. Análise Espacial de Dados Geográficos. Brasília: EMBRAPA, 2004.

FUNDAÇÃO INSTITUTO BRASILEIRO DE GEOGRAFIA E ESTATÍSTICA. Censo demográfico 2000. Rio de Janeiro: Fundação Instituto Brasileiro de Geografia e Estatística. 2001.

HADDAD, M.; NEDOVIC-BUDIC, Z. Using Spatial Statistics to Analyze Intra-urban Inequalities and Public Intervention in São Paulo, Brazil. Journal of Human Development, v. 7, n. 1, p. 85109, 2006.

HENNINGER, N.; SNEL, M. Where is the poor? Experience with the development and use of poverty maps, 2002. Disponível em 〈http://archive.wri.org/publication_detail.cfm?pubid=3758>. Acesso em: 08 jan. 2010..

INSTITUTO DE PESQUISA E PLANEJAMENTO URBANO DE CURITIBA. Resumo gerencial - indicadores de qualidade de vida - 2000. Curitiba: Instituto de Pesquisa e Planejamento Urbano de Curitiba, 2004.

NEVES, M. C. Et al. Análise exploratória espacial de dados sócio-econômicos de São Paulo, 2000. Disponivel em: 〈http://www.dpi.inpe.br/gilberto/papers/marcos_gisbrasil2000.pdf $>$. Acesso em: 08 jan. 2010.

OLIVER, S.; GOTWAY, C. A. Statistical methods for spatial data analysis. London: Chapman \& Hall/CRC, 2005.

PERÉZ G. J. Dimension Espacial de la probreza en Colombia, 2005. Disponível em: < http://www.banrep.gov.co/docum/Pdf-econom-region/Documentos/DTSER-54.pdf>. Acesso em: 06 jan. 2010.

PETRUCCI, A. ; SALVATI, N. ; SEGHIERI, C. Autologistic regression model for poverty mapping and analysis. Metodološki Zvezky. v. 1, n. 1, p. 225-234, 2004. 
SLIWIANY, R. M. Sociometria, como medir a qualidade de vida e projetos sociais. Petrópolis: Vozes, 1997.

SPOSATI, A. Mapa da exclusão/inclusão social da cidade de São Paulo. São Paulo: EDUC, 1996. 